\title{
Coherent single-spin source based on topological insulators
}

\author{
Yanxia Xing, ${ }^{1,2, *}$ Zhong-liu Yang, ${ }^{1}$ Qing-feng Sun, ${ }^{3,4}$ and Jian Wang ${ }^{2, \dagger}$ \\ ${ }^{1}$ Department of Physics, Beijing Institute of Technology, Beijing 100081, China \\ ${ }^{2}$ Department of Physics and the Center of Theoretical and Computational Physics, The University of Hong Kong, \\ Pokfulam Road, Hong Kong, China \\ ${ }^{3}$ International Center for Quantum Materials, Peking University, Beijing 100871, China \\ ${ }^{4}$ Collaborative Innovation Center of Quantum Matter, Beijing, China
}

(Received 1 April 2014; revised manuscript received 3 July 2014; published 27 August 2014)

\begin{abstract}
We report on the injection of quantized pure spin current into quantum conductors. In particular, we propose an on-demand single-spin source generated by periodically varying the gate voltages of two quantum dots that are connected to a two-dimensional topological insulator via tunneling barriers. Due to the nature of the helical states of the topological insulator, one or several spin pairs can be pumped out per cycle giving rise to a pure quantized alternating spin current. Depending on the phase difference between two gate voltages, this device can serve as an on-demand single-spin emitter or single-charge emitter. Again, due to the helicity of the topological insulator, the single-spin emitter or charge emitter is dissipationless and immune to disorder. The proposed single-spin emitter can be an important building block of future spintronic devices.
\end{abstract}

DOI: 10.1103/PhysRevB.90.075435

PACS number(s): 73.23.-b, 72.80.-r, 72.25.Mk

\section{INTRODUCTION}

Traditional electronics is based on the flow of charge where the spin of the electron is ignored. The emerging technology of spintronics [1] will explore the spin degree of freedom such that the flow of spin, in addition to charge, will be used for electronic applications [2]. Many applications in spintronics have been demonstrated, such as the giant magnetoresistive effect [3], the spin injection across a magnetic-nonmagnetic interface [4], and optical manipulation of spin degrees of freedom [5]. The spin degree of freedom can also be used to process quantum information [6]. It is well known that the quantum bit or qubit is one of the basic building blocks for quantum information science. A large variety of candidate qubit systems have been proposed such as photonic qubits [7], electron qubits [8], and so on.

Recently, an on-demand coherent single-electron source has been produced experimentally [9] and later studied theoretically [10]. By applying ac gate voltage, a periodic sequence of single-electron emission and absorption on nanoseconds generates a quantized ac current. The single-electron transfer between two distant quantum dots (QDs) has also been demonstrated, which paves the way for single-electron circuitry [11]. This single-electron source can also be used as a qubit in ballistic conductors, which is an important step towards $2 \mathrm{DEG}$ quantum computers. The big challenge in the realization of quantum computers is to identify qubits with long coherence time. From this point, a spin qubit seems to be the ideal candidate [12]. This is because the spin of electron is weakly coupled to the environment compared with the charge degree of freedom, therefore, the quantum coherence can be maintained at much longer time scales [13]. It is therefore important to study the transport properties of an on-demand coherent single-spin source, which can be used as spin qubit.

The topological insulator (TI), a new state of matter, has attracted a lot of theoretical and experimental attention

\footnotetext{
*xingyanxia@bit.edu.cn

†jianwang@hkusua.hku.hk.
}

[14-16]. The TI has an insulating energy gap in the bulk that behaves like the general insulator, but it has exotic gapless metallic states on its edges or surface. The TI was first predicted in two-dimensional (2D) systems, e.g., graphene [17], $\mathrm{HgTe} / \mathrm{CdTe}$ quantum wells [18], or InAs/GaSb quantum wells [19], and has been generalized in 3D [20] and confirmed experimentally [21]. The 2D TI has gapless helical edge states and exhibits the quantum spin Hall effect while in 3D TI the conducting state is a helical surface state. This helical edge or surface states are topologically protected and are robust against all time-reversal-invariant impurities. Many interesting physical phenomena have been predicted including Majorana fermions [22], spin pumping or time-dependent spin injection [23], magneto-optical Kerr and Faraday effects [24], and so on.

In this paper, we report on the injection of quantized pure spin current into quantum conductors. In particular, using the concept of parametric pumping [25-27], we study an on-demand single-spin source generated by periodically varying the gate voltage applied at two QDs that are connected to a two-dimensional TI via tunneling barriers. Due to the nature of helical edge state of TI, each QD will generate a fully spin polarized ac current localized near the edge of TI, while the direction of the current is controlled by the phase of the gate voltage. As a result of the time reversal symmetry, either pure charge current or pure spin current can be generated depending on the phase between two gate voltages of QDs. When the phase difference is $\pi$, there is a quantized ac spin current with $n$ spin per cycle pumped out giving rise to a single-spin emitter, where $n$ is an integer. When the phase difference is zero, a spin unpolarized quantized ac charge current is generated with $n$ charge per cycle. We emphasize that the generated spin current has no accompanying charge current and thus is a pure ac spin source. To study the quantized spin emitter, a quantum transport theory for time-dependent pumped current using the nonequilibrium Green's function method in the adiabatic regime is developed. Numerical calculations show that the quantized spin current is independent of the geometry of QD. Also, it is robust against the weak disorders. 


\section{MODEL}

In a 2D TI, when the Fermi energy is inside the energy gap, electrons can only be transported through the unidirectional spin locked edge state. In our study, the 2D TI ribbon is coupled with two QDs at the upper and lower edges whose energy levels are controlled by gate voltages $V_{t}(t)$ and $V_{b}(t)$, respectively [see Figs. 1(a) and 1(c), respectively]. We assume that the amplitudes of two gate voltages are the same but with a phase difference $\phi$. As we will discuss below, when $\phi=0$, the system behaves like a coherent single-electron source, while when $\phi=\pi$ an alternating quantized spin current is generated, i.e., the system is an on-demand single-spin emitter without an accompanying electric current.

Due to the variation of the gate voltage, the current with spin up and down [shown in Figs. 1(a) and 1(c), respectively] can be pumped out. In the following discussion, we first focus on the top QD and spin-up transport [see Fig. 1(a)]. Note that due to the nature of the helical state there is no spin-up electron or hole from the top QD to the left lead. Assuming that at $t=0, U_{t}=-e V_{0}$ is minimum, the energy level of QD is below the Fermi level and is completely filled, i.e., filled with two electrons of opposite spin. In the first half-period, $V_{t}(t)$ decreases from $V_{0}$ to $-V_{0}$, the energy levels of QD shift upward from $-U_{0}$ to $U_{0}$ so that electrons flow out of the QD and into the right lead [see the blue solid arrow in Fig. 1(a)]. In the second half period, the energy levels shift downwards from $U_{0}$ to $-U_{0}$, consequently, the holes are flowing out of the QD to the right lead [see the blue hollow arrow in Fig. 1(a)]. It means under certain conditions, only one electron (hole) with spin up is pumped out from the top QD into the right lead giving rise to an alternating quantized spin-polarized charge current. In the meantime, due to the time reversal symmetry, a spin-down electron (hole) is pumped out of the QD to the left lead in the first (second) half-period [see the blue arrows in Fig. 1(a)]. We emphasize that if there were no bottom QD

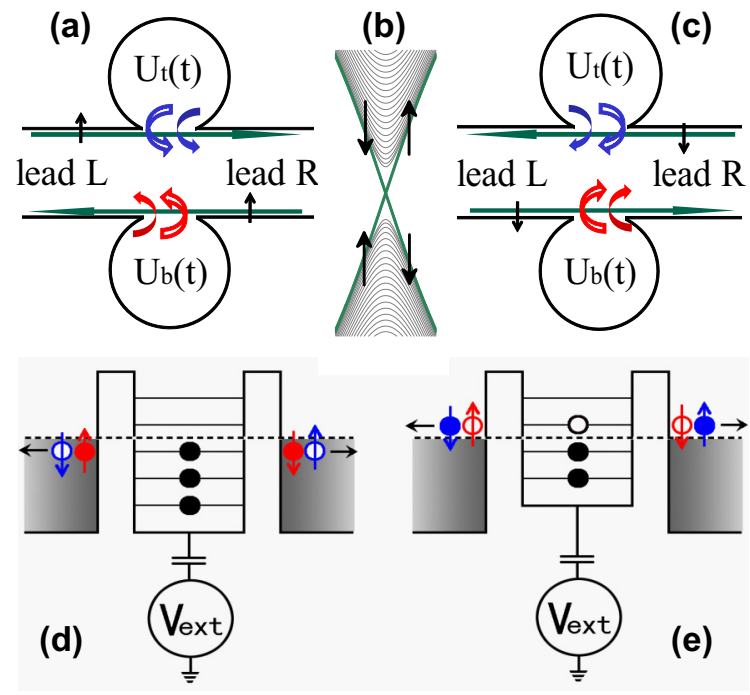

FIG. 1. (Color online) (a) and (c) Schematic plot of pumped current in the whole period for the spin up and spin down. (b) Band structure of TI. Combining (a) and (c), we can get (d) and (e) that depict the generation of pumped pure spin current in the first and second half-periods, respectively. there would be no spin-up (down) current in the left (right) lead. Now, we consider the independent bottom QD in which the electrons and holes are pumped out similar to the top QD [see the red arrows in Figs. 1(a) and 1(c)]. If the gate voltages of the upper and lower QD are out of phase (such as $\left.\phi_{b}=0, \phi_{t}=\pi\right)$, the spin pair composed of an electron and a hole with opposite spins will be pumped into the left and right leads in each half-period. In this case, the net charge current is zero. However, there is a pure quantized spin current from the left to the right lead in the first half-period that reverses its sign in the second half-period as shown in Figs. 1(d) and 1(e). If the phase difference between two gate voltages is zero, a charge pair or a hole pair with opposite spin will be pumped out leading to an alternating quantized charge current.

\section{THEORY}

The working principle of a coherent single-spin emitter (SSE) is based on a 2D TI with a helical spin texture present in momentum space. In order to highlight the functional mechanism of SSE and capture its salient features, we use a modified Dirac model with a quadratic corrections $k^{2} \sigma_{z}$, which has been shown to have similar properties [28] as $\mathrm{HgTe} / \mathrm{CdTe}$ but with symmetric conductance and valance bands. The Hamiltonian is given by $H(k)=\left[H_{\uparrow}(\mathbf{k})+H_{\downarrow}(\mathbf{k})\right]$, where

$$
\begin{aligned}
H_{\uparrow}(\mathbf{k})= & H_{\downarrow}^{*}(-\mathbf{k})=A\left(\mathbf{k}_{x} \sigma_{x}-\mathbf{k}_{y} \sigma_{y}\right) \\
& +(m+B \mathbf{k} \cdot \mathbf{k}) \sigma_{z}+\epsilon(r) \sigma_{0} .
\end{aligned}
$$

Here, $\sigma_{x, y, z}$ are Pauli matrices presenting the pseudospin formed by $s, p$ orbitals and $\sigma_{0}$ is a unitary $2 \times 2$ matrix. The individual spin-up Hamiltonian $H_{\uparrow}$ and spin-down Hamiltonian $H_{\downarrow}$ are time reversal symmetric to each other, and can be calculated independently. To carry out numerical calculations, the tight-binding Hamiltonian in a square lattice is employed, which is written as $[28,29]$

$$
\begin{aligned}
H_{\uparrow}= & \sum_{\mathbf{i}} d_{\mathbf{i}}^{\dagger}\left[\epsilon_{\mathbf{i}} \sigma_{0}+(m-4 t) \sigma_{z}\right] d_{\mathbf{i}} \\
& +\sum_{\mathbf{i}}\left[d_{\mathbf{i}}^{\dagger}\left(t \sigma_{z}-i \frac{A}{2 a} \sigma_{x}\right) d_{\mathbf{i}+\delta_{x}}\right. \\
& \left.+d_{\mathbf{i}}^{\dagger}\left(t \sigma_{z}+i \frac{A}{2 a} \sigma_{y}\right) d_{\mathbf{i}+\delta_{y}}\right]+ \text { H.c. }
\end{aligned}
$$

where $\epsilon_{\mathbf{i}}$ is a random on-site potential, which is uniformly distributed in the region $[-w / 2, w / 2]$ and $\mathbf{i}=\left(\mathbf{i}_{x}, \mathbf{i}_{y}\right)$ is the index of the discrete site in the unit vectors of the square lattice with the lattice constant $a=5 \mathrm{~nm} . d_{\mathbf{i}}=\left[d_{s, \mathbf{i}}, d_{p, \mathbf{i}}\right]^{T}$ with $T$ denoting transpose, $d_{s(p), \mathbf{i}}$ and $d_{s(p), \mathbf{i}}^{\dagger}$ are the annihilation and creation operators for an $s(p)$ orbital at site i. Here, $A / 2 a=$ $1.35 t, m=-0.35 t$, and $t=B / a^{2}=27.5 \mathrm{meV}$ denote the nearest-neighbor coupling strength.

To calculate the time-dependent pumped current, it is convenient to examine the adiabatic regime. In the lowfrequency limit, the system is nearly in equilibrium and the time-dependent pumping parameters are adiabatically added to the Hamiltonian [25]. The particle distribution in the scattering 
region at any instant is given by

$$
N_{\sigma}(\mathbf{i}, t)=-i \int \frac{d E}{2 \pi}\left[\mathbf{G}_{\sigma}^{<}(E,\{\mathbf{U}(t)\})\right]_{\mathbf{i i}},
$$

where $\sigma=\uparrow / \downarrow$ or \pm 1 denotes spin up and spin down and $\mathbf{U}(t)$ is the pumping potential. Since there is no external driving force, the left and right leads have the same Fermi energy. From the fluctuation-dissipation theorem, $\mathbf{G}_{\sigma}^{<}=\left(\mathbf{G}_{\sigma}^{a}-\mathbf{G}_{\sigma}^{r}\right) f(E)$ with $f(E)$ being the equilibrium distribution function for the left and right leads, we can get the total particle number in the scattering region:

$$
N_{\sigma}(t)=-i \int \frac{d E}{2 \pi} \operatorname{Tr}\left[\left(\mathbf{G}_{\sigma}^{a}-\mathbf{G}_{\sigma}^{r}\right) f(E)\right],
$$

where $\mathbf{G}_{\sigma}^{r}(E,\{\mathbf{U}(t)\})=\left[E \mathbf{I}-\mathbf{H}_{\sigma}-\Sigma_{\sigma}^{r}(E)-\mathbf{U}(t)\right]^{-1}, \Sigma_{\sigma}^{r}=$ $\Sigma_{L, \sigma}^{r}+\Sigma_{R, \sigma}^{r}$ is the self-energy from the semi-infinite left and right leads, and $\mathbf{U}$ is a diagonal matrix with $\mathbf{U}=\sum_{i=b / t} U_{i} \boldsymbol{\Delta}_{i}$, where $U_{b / t}=-e V_{b / t}$ is the pumping parameter induced by the gate voltage $V_{b / t}$ added in the bottom or top QD and $\boldsymbol{\Delta}_{b / t}$ is the pumping potential profile, which is a matrix labeling where is the pumping potential, while the pumping parameter $U_{b / t}$ denotes the magnitude of the pumping potential. Due to the variation of pumping parameters $U_{b / t}(t)$, the total pumped particle current into all contacts is [26]

$$
d N_{\sigma}(t) / d t=-i \int \frac{d E}{2 \pi} f(E) \sum_{i} \partial_{U_{i}} \operatorname{Tr}\left(\mathbf{G}_{\sigma}^{a}-\mathbf{G}_{\sigma}^{r}\right) d U_{i} / d t .
$$

From the Dyson equation $\mathbf{G}_{\sigma}^{r}=\mathbf{G}_{\sigma}^{r, 0}+\mathbf{G}_{\sigma}^{r, 0} \mathbf{U} \mathbf{G}_{\sigma}^{r}$ with $\mathbf{G}_{\sigma}^{r, 0}=$ $\left[E \mathbf{I}-\mathbf{H}_{\sigma}-\Sigma_{\sigma}^{r}\right]^{-1}$, we obtain

$$
\partial_{U_{i}} \operatorname{Tr}\left[\mathbf{G}_{\sigma}^{r / a}\right]=\operatorname{Tr}\left[\mathbf{G}_{\sigma}^{r / a} \Delta_{i} \mathbf{G}_{\sigma}^{r / a}\right]=-\partial_{E} \operatorname{Tr}\left[\mathbf{G}_{\sigma}^{r / a} \boldsymbol{\Delta}_{i}\right] .
$$

Note that bold letters such as for the Green's function $\mathbf{G}_{\sigma}^{r}$, self-energy $\Sigma_{\sigma}^{\mathbf{r}}$, and potential profile $\boldsymbol{\Delta}$ are all matrices that do not commute but can be rotated under the trace operator. Using $G_{\sigma}^{a}-G_{\sigma}^{r}=i G_{\sigma}^{r} \Gamma_{\sigma} G_{\sigma}^{a}$ and integrating Eq. (3) by part, we get the total instantaneous particle current from all leads:

$$
d N_{\sigma} / d t=\sum_{i} \operatorname{Tr}\left[\mathbf{G}_{\sigma}^{r}\left(\sum_{\alpha} \boldsymbol{\Gamma}_{\alpha, \sigma}\right) \mathbf{G}_{\sigma}^{a} \boldsymbol{\Delta}_{i}\right] d U_{i} / d t,
$$

where $\boldsymbol{\Gamma}_{\alpha, \sigma}$ is the coupling strength between the scattering region and the lead $\alpha$ for the electron with spin $\sigma$. Obviously, the above equation gives the current partition into each lead $\alpha$. For the pumped charge and spin current, we have

$$
\begin{aligned}
& d Q_{\alpha} / d t=-e \sum_{\sigma} d N_{\sigma, \alpha} / d t, \\
& d S_{\alpha} / d t=(\hbar / 2) \sum_{\sigma} \sigma d N_{\sigma, \alpha} / d t,
\end{aligned}
$$

where $d N_{\sigma, \alpha} / d t=\sum_{i} \operatorname{Tr}\left[\mathbf{G}_{\sigma}^{r} \boldsymbol{\Gamma}_{\alpha, \sigma} \mathbf{G}_{\sigma}^{a} \boldsymbol{\Delta}_{i}\right] d U_{i} / d t$. In order to get a better understanding of the SSE, we integrate Eq. (5) to get the total spin emitted in half-period,

$$
S_{\alpha}=\frac{\hbar}{2} \int d t \operatorname{Tr} \sum_{\sigma} \sigma\left[\mathbf{G}_{\sigma}^{r}(E, \mathbf{U}) \boldsymbol{\Gamma}_{\alpha, \sigma} \mathbf{G}_{\sigma}^{a}(E, \mathbf{U}) d \mathbf{U} / d t\right] .
$$

In the whole period, the time averaged spin current is zero, since $d \mathbf{U}$ is zero in a period.

\section{NUMERICAL RESULTS}

In the numerical calculation, we discretize the scattering region shown in the inset of Fig. 3 to obtain a tight-binding Hamiltonian which can be used to construct the retarded Green's function. For convenience, we set the temperature to zero. We expect that our results remain valid at low temperatures, e.g., a few degrees Kelvin. We also fix the Fermi energy of the left and right leads as $E_{F}=0$. The side-coupled QDs, modeled by the modified Dirac model, have broadening energy levels $\epsilon_{ \pm n}$ that are symmetrically distributed with respect to a reference energy $E_{0}$. In equilibrium, i.e., $V_{b / t}=0$, $E_{0}=E_{F}=0$. When the tuning gate voltage is $V_{b / t}, \epsilon_{ \pm n}$ is shifted. For the QD, the level spacing $\Delta \epsilon$ between two nearest energy levels is around $0.2 t \approx 5 \mathrm{meV}$. For this level spacing, the system is in the adiabatic regime for the ac signal (a few $\mathrm{GHz}$ ) used in most of the experiments since $\Delta \epsilon>>\hbar \omega$. In the adiabatic regime, the energy levels of QDs respond to the ac gate voltage $V_{b / t}(t)$ instantaneously. We assume that $V_{b / t}(t)=V_{0} \pm V(t)$ (with opposite phase), where $V_{0}$ is the static gate voltage that is used to tune the reference energy $E_{0}$.

In Fig. 2, we plot the instantaneous pumped spin current in the whole period for the different reference energies $E_{0}$. We define the positive current as the current flowing right. It is found that the pump current peaks when the Fermi energy sweeps through the energy levels of QD. For $E_{0}=0$, when $\tau$ changes from 0 to $T / 2, E_{F}$ crosses over the two levels $\epsilon_{ \pm 1}$. So, two peaks appear in Figs. 2(c) and 2(d). Note that due to the coupling of the leads, the energy levels of QD, $\epsilon_{ \pm n}$, are slightly different from the isolated QD, $\epsilon_{ \pm n}^{\text {iso }}$. For example, $\epsilon_{ \pm 1}^{\text {iso }}= \pm 0.19 t$ and $\epsilon_{ \pm 1}= \pm 0.15 t$, and so on. When $E_{0}=0.15 t$, the $E_{F}$ is in line with $\epsilon_{-1}$ at $t=T / 2$, for the small amplitude $V_{b / t}$, the Fermi energy scans over only the level of $\epsilon_{-1}$ in a half-period, then one peak appears in Figs. 2(a) and 2(b). Using Eq. (7), we can integrate the spin current and get the pumped spin pairs in a half-period. In Figs. 2(a), 2(b), 2(c), and $2(\mathrm{~d})$, the number of the spin pairs in a half-period is 1.015 ,
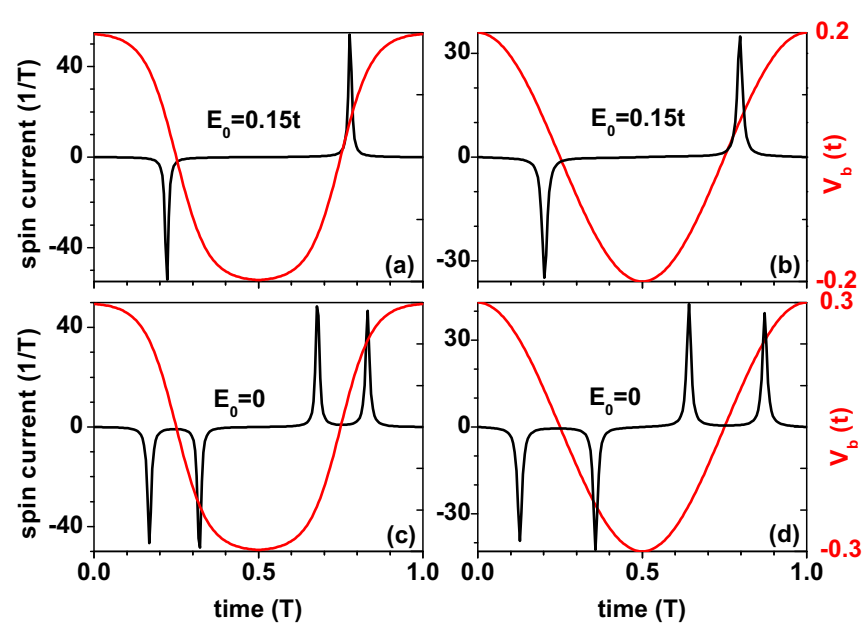

FIG. 2. (Color online) Pumped current driven by a general ac signal [gray line in (a) and (c)] and harmonic signal [gray line in (b) and (d)]. The red line is the profile of the gate voltage $V_{b}(t)$. The width of the lead $W=40 a$. The diameter of the circular QD is $D=21 a$. The contact width of QPC $L=3 a$. 


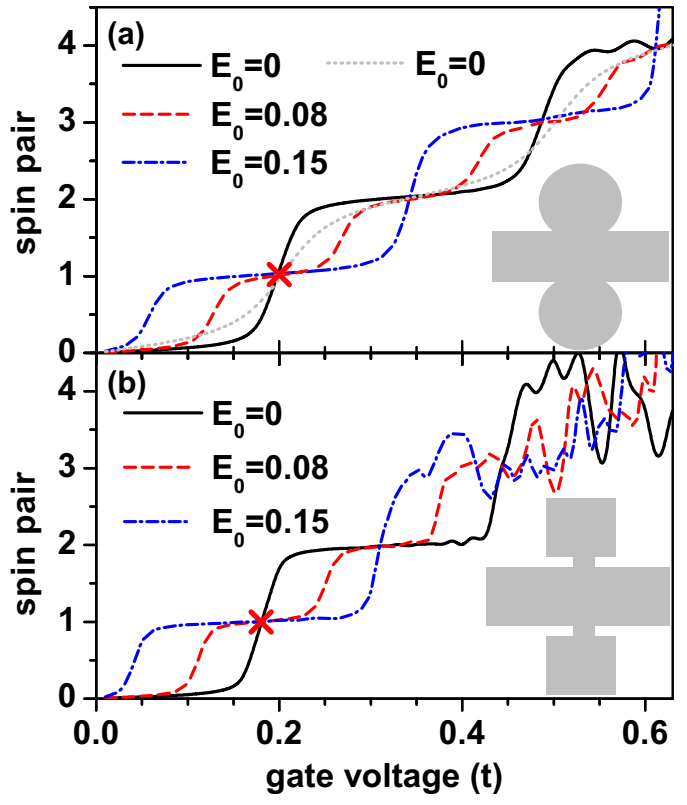

FIG. 3. (Color online) The number of spin pairs pumped out of QD in the first half-period vs the magnitude of the ac gate voltage. Different curves correspond to different $E_{0}$. The diameter of the circular QD is $D=21 a$ and the contact width $L=3 a$ (thick lines) and $L=7 a$ (thin gray dotted line). The size of square QD is $20 a \times$ $20 a$ and the contact width $L=7 a$.

$1.015,1.957,1.957$, respectively, very close to the quantized number 1 and 2. Figure 2 also shows that various forms of ac signals such as inharmonic ac signal and harmonic signal can also generate different instantaneous currents. However, they all pump out exactly one quantum spin pair in a half-period. So this scheme can be used as a general spin source in spintronic devices.

In the following, we will show that a single-spin source can be realized in all kinds of QDs with different geometric shapes. In Fig. 3, we plot the quantized spin pairs accumulated in a half-period. We consider QDs with two geometries: circular [panel (a)] and square shaped [panel (b)]. For different $E_{0}$, there are three representative configurations of quantization: when $E_{F}=0$ is in line with $E_{0}, V_{b, t}$ scans through an even number of energy levels in a half-period, and the number of spin pairs pumped out is $0,2,4, \ldots$ (see black solid lines in Fig. 3). When $E_{F}$ is roughly in line with $\epsilon_{ \pm n}$, such as $\epsilon_{-1}$ $\left(E_{0}=0.15 t\right)$, the number of pumped spin pairs is $1,3,5 \ldots$ (see dash dotted lines in Fig. 3), otherwise, the number of spin pairs pumped out is an integer $1,2,3 \ldots$. Furthermore, comparing the black solid line with the gray dotted line in Fig. 3(a), we can see that the weaker the coupling between $\mathrm{TI}$ and QD, the longer the quantized spin plateau is. This is because when the coupling is weak, the bandwidth is small, then it is more easier for electrons to escape completely within the voltage interval $\left[-V_{0}, V_{0}\right]$.

In Fig. 4, we plot the total spin on and before the first quantized plateau (the red cross in Fig. 3) vs on-site disorder strengths for different $E_{0}$. For $E_{0}=0$, the red cross (in Fig. 3) is just in between two quantized plateaus. This is similar to the quantum Hall effect that when the Fermi level is very close to

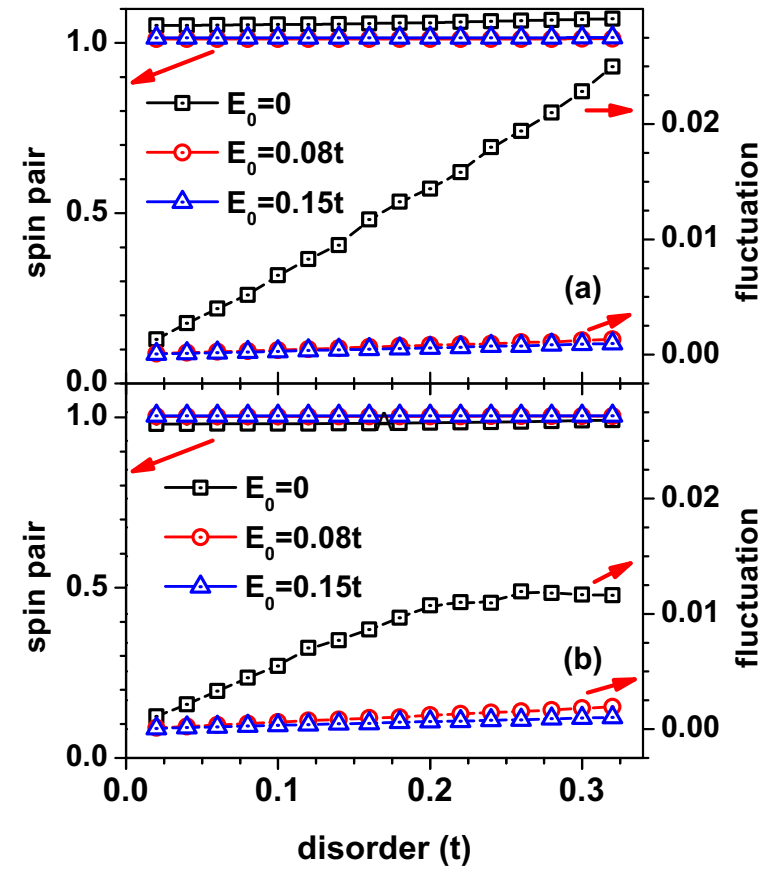

FIG. 4. (Color online) The average number of spin pairs and its fluctuation at the gate voltage marked with a red cross in Fig. 3 vs disorder strengths for different $E_{0}$. (a) and (b) correspond to the circular and square shaped QDs.

the discrete bulk Landau level a small disorder will make the edge state relax to the bulk state, while when the Fermi level is away from the Landau level the quantized plateau is very robust against disorder. When $E_{0}=0.08$ and 0.15 , they are in the middle of the quantized plateau and hence the helical states are very robust. Importantly, it is shown that although fluctuations of the quantized spin can be large, e.g., $E_{0}=0$, the averaged values of quantized spin are hardly changed by disorder, especially for the quantized values on the quantum plateaus. This means that this spin emitter is robust against the disorder scattering. We have not examined the effect of spin dependent disorder, which is known to be present in HgTe quantum wells [30]. This may lead to a departure of the quantized spin and charge pumping.

In conclusion, we have proposed a single-spin emitter that is driven by two ac gate voltages. Due to the helical feature of TI, an alternating pure spin current with integer number of spin pairs per cycle can be generated. Importantly, by tuning the phase difference between two gate voltages, either an ac quantized spin current or an ac quantized charge current can be pumped out. Our numerical results show that this quantized single-spin emitter is robust against disorder and variation of device shapes.

\section{ACKNOWLEDGMENTS}

We gratefully acknowledge the financial support from from NSF-China under Grant (Nos. 11174032 and 11374246), NBRP of China (2012CB921303), and a RGC Grant (HKU 705212P) from the Government of HKSAR. 
[1] I. Žutić, J. Fabian, and S. Das Sarma, Rev. Mod. Phys. 76, 323 (2004).

[2] S. A. Wolf, D. D. Awschalom, R. A. Buhrman, J. M. Daughton, S. von Molnár, M. L. Roukes, A. Y. Chtchelkanova, and D. M. Treger, Science 294, 1488 (2001); G. A. Prinz, ibid. 282, 1660 (1998).

[3] M. N. Baibich, J. M. Broto, A. Fert, F. Nguyen Van Dau, F. Petroff, P. Etienne, G. Creuzet, A. Friederich, and J. Chazelas, Phys. Rev. Lett. 61, 2472 (1988); R. Meservey and P. M. Tedrow, Phys. Rep. 238, 173 (1994).

[4] G. Schmidt, D. Ferrand, L. W. Molenkamp, A. T. Filip, and B. J. van Wees, Phys. Rev. B 62, R4790(R) (2000); C.-M. Hu, J. Nitta, A. Jensen, J. B. Hansen, and H. Takayanagi, ibid. 63, 125333 (2001).

[5] J. M. Kikkawa, I. P. Smorchkova, N. Samarth, and D. D. Awschalom, Science 277, 1284 (1997); F. Pezzoli, F. Bottegoni, D. Trivedi, F. Ciccacci, A. Giorgioni, P. Li, S. Cecchi, E. Grilli, Y. Song, M. Guzzi, H. Dery, and G. Isella, Phys. Rev. Lett. 108, 156603 (2012).

[6] E. Nagali, F. Sciarrino, F. De Martini, L. Marrucci, B. Piccirillo, E. Karimi, and E. Santamato, Phys. Rev. Lett. 103, 013601 (2009); M. Leijnse and K. Flensberg, ibid. 107, 210502 (2011).

[7] A. Shaham and H. S. Eisenberg, Phys. Rev. A 83, 022303 (2011); R. Ikuta, H. Kato, Y. Kusaka, S. Miki, T. Yamashita, H. Terai, M. Fujiwara, T. Yamamoto, M. Koashi, M. Sasaki, Z. Wang, and N. Imoto, ibid. 87, 010301(R) (2013).

[8] E. Knill, R. Laflamme, and G. J. Milburn, Nature (London) 409, 46 (2001); G. Barcza, Ö. Legeza, K. H. Marti, and M. Reiher, Phys. Rev. A 83, 012508 (2011).

[9] G. Fève, A. Mahé, J.-M. Berroir, T. Kontos, B. Plaçais, D. C. Glattli, A. Cavanna, B. Etienne, and Y. Jin, Science 316, 1169 (2007); C. Mora and K. L. Hur, Nat. Phys. 6, 697 (2010).

[10] M. Moskalets, P. Samuelsson, and M. Buttiker, Phys. Rev. Lett. 100, 086601 (2008).

[11] S. Hermelin, S. Takada, M. Yamamoto, S. Tarucha, A. D. Wieck, L. Saminadayar, C. Bäuerle, and T. Meunier, Nature (London) 477, 435 (2011); R. P. G. McNeil, M. Kataoka, C. J. B. Ford, C. H. W. Barnes, D. Anderson, G. A. C. Jones, I. Farrer, and D. A. Ritchie, ibid. 477, 439 (2011).

[12] D. Loss and D. P. DiVincenzo, Phys. Rev. A 57, 120 (1998).

[13] B. Trauzettel, D. V. Bulaev, D. Loss, and G. Burkard, Nat. Phys. 3, 192 (2007).

[14] J. E. Moore, Nature (London) 464, 194 (2010).

[15] M. Z. Hasan and C. L. Kane, Rev. Mod. Phys. 82, 3045 (2010).
[16] X.-L. Qi, T. L. Hughes, and S.-C. Zhang, Phys. Rev. B 78, 195424 (2008); X.-L. Qi and S.-C. Zhang, Rev. Mod. Phys. 83, 1057 (2011).

[17] C. L. Kane and E. J. Mele, Phys. Rev. Lett. 95, 226801 (2005); 95, 146802 (2005).

[18] B. A. Bernevig, T. L. Hughes, and S.-C. Zhang, Science 314, 1757 (2006); M. König, S. Wiedmann, C. Brüne, A. Roth, H. Buhmann, L. W. Molenkamp, X.-L. Qi, and S.-C. Zhang, ibid. 318, 766 (2007).

[19] C. Liu, T. L. Hughes, X.-L. Qi, K. Wang, and S.-C. Zhang, Phys. Rev. Lett. 100, 236601 (2008); I. Knez, R.-R. Du, and G. Sullivan, ibid. 107, 136603 (2011); 109, 186603 (2012).

[20] L. Fu, C. L. Kane, and E. J. Mele, Phys. Rev. Lett. 98, 106803 (2007); H. Zhang, C.-X. Liu, X.-L. Qi, X. Dai, Z. Fang, and S.-C. Zhang, Nat. Phys. 5, 438 (2009).

[21] D. Hsieh, D. Qian, L. Wray, Y. Xia, Y. S. Hor, R. J. Cava, and M. Z. Hasan, Nature (London) 452, 970 (2008).

[22] L. Fu and C. L. Kane, Phys. Rev. Lett. 100, 096407 (2008); 102, 216403 (2009).

[23] R. Citro, F. Romeo, and N. Andrei, Phys. Rev. B 84, 161301(R) (2011); A. Inhofer and D. Bercioux, ibid. 88, 235412 (2013); P. P. Hofer and M. Büttiker, ibid. 88, 241308(R) (2013); F. Dolcini, ibid. 83, 165304 (2011); D. Ferraro, G. Dolcetto, R. Citro, F. Romeo, and M. Sassetti, ibid. 87, 245419 (2013).

[24] W.-K. Tse and A. H. MacDonald, Phys. Rev. Lett. 105, 057401 (2010).

[25] P. W. Brouwer, Phys. Rev. B 58, R10135 (1998).

[26] Y. Wei and J. Wang, Phys. Rev. B 66, 195419 (2002).

[27] D. J. Thouless, Phys. Rev. B 27, 6083 (1983); Y. Wei, J. Wang, and H. Guo, ibid. 62, 9947 (2000); J. E. Avron, A. Elgart, G. M. Graf, and L. Sadun, ibid. 62, R10618 (2000); B. Wang, J. Wang, and H. Guo, ibid. 65, 073306 (2002); M. G. Vavilov, L. DiCarlo, and C. M. Marcus, ibid. 71, 241309 (2005); L. Arrachea, ibid. 72, 125349 (2005); J. Splettstoesser, M. Governale, J. König, and R. Fazio, ibid. 74, 085305 (2006).

[28] Y. Xing, L. Zhang, and J. Wang, Phys. Rev. B 84, 035110 (2011).

[29] J. Li, R.-L. Chu, J. K. Jain, and S.-Q. Shen, Phys. Rev. Lett. 102, 136806 (2009); H. Jiang, L. Wang, Q.-F. Sun, and X. C. Xie, Phys. Rev. B 80, 165316 (2009).

[30] M. Köenig, H. Buhmann, L. W. Molenkamp, T. Hughes, C.-X. Liu, X.-L. Qi, and S.-C. Zhang, J. Phys. Soc. Jpn. 77, 031007 (2008); D. Rothe, R. W. Reinthaler, C.-X. Liu, L. W. Molenkamp, S.-C. Zhang, and E. M. Hankiewicz, New J. Phys. 12, 065012 (2010). 